\title{
Reliability in Multicast Services and Protocols ; A Survey
}

\author{
Christophe Diot \\ INRIA Sophia Antipolis \\ 2004 Route des Lucioles, BP 93, 06902 Sophia Antipolis, France \\ Ph. (33) 93657756 ; Fax. (33) 93657765 ; \\ e.mail :christophe.diot@sophia.inria.fr
}

\begin{abstract}
Multipoint communication allows information transfer within a group of peer entities and is becoming more and more relevant in distributed environments. For distributed or replicated data, it ensures an efficient communication without overloading the network. Multicast is defined as a subset of multipoint where a single sender transmits data to a group of receivers. Several classes of multicast services have been defined to respond to application requirements in terms of efficiency, reliability, and performance. This paper surveys protocol functions and mechanisms for reliable multicast data transmission. A multicast protocol basically consists of a three phase protocol (data transmission, acknowledgment, and validation), which can be implemented in various ways. This paper characterizes reliability in the studied protocols. Functionalities and implementation mechanisms are analyzed. The paper concludes with a statement on current developments in multicast Transport protocol and service definitions.
\end{abstract}

Keywords

Communication Network, Multicast Protocol, Reliability, Error Control, Flow Control.

\section{O INTRODUCTION}

The most urgent demand for enhanced communication protocols comes from multipoint applications. Multipoint Applications are applications where more than two users (these users define a "group") may want to exchange information ; in contrast, point to point applications involve only two users. Today, the communication environment is mainly point to point. The OSI Reference Model is point to point. TCP/IP, the Internet standard, is a point to point protocol ; however, IP (and UDP) have received some modifications to support multipoint commu- 
nications [Deering 89]. Consequently, applications that require multipoint communication generally use non reliable multicast transmission over a UDP/IP stack. Examples of public domain applications using such multicast support are IVS, for video-conferencing [Turletti 93], and WB, a distributed white-board [Jacobson 93].

Such enhancements are sufficient for many applications which can be satisfied by a connectionless multicast communication support. The problem is now to increase the reliability of multipoint communication providing connection oriented services. The multiplication of multimedia applications, associated with new high speed networks based on ATM technology (which also does not provide any facility for multipoint communication), is driving the need for reliable multipoint communication stacks in the very near future. This paper characterizes reliability in multicast transmission. It is based on a survey of existing protocols.

Section 1 gives a general presentation of multipoint communication (defined as an $\mathrm{N}$ to $\mathrm{N}$ communication). Then, it focuses on a subset of multipoint that is multicast (information transfer from a single sender to $\mathrm{N}$ receivers). The need for $\mathrm{N}$ to $\mathrm{N}$ communication exists, but it is marginal, and very difficult to provide. The analysis of applications that need multipoint communication support shows that multicast is sufficient in most of the cases (see section 1.1). The section concludes with a presentation of the protocols (from the Transport to the LLC Layer) we have selected to support our discussion on reliability in multicast transmission. In section 2 protocols are characterized by the mechanisms used to provide reliability in multicast transmission. This analysis is based on application requirements that can justify reliability. In section 3, mechanisms and algorithms used to implement the reliability are analyzed. An overview of multicast protocol characteristics which are not directly linked to reliability (concentration, group management, multicast routing in WAN) is given at the end of the section. The paper concludes with a survey of current international activities around new multicast protocol and service definitions.

\section{AN INTRODUCTION TO MULTICAST}

\subsection{Definitions}

In distributed systems, cooperating processes communicate with each other by exchanging messages. The easiest way to communicate consists of a single source process sending a message to a single destination process; this achieves a unicast communication (or point to point communication). Other more sophisticated communications exist, involving a set of cooperating processes. In order to use a coherent definition in this paper, and because the literature does not provide a unique definition, we will call multipoint a $\mathrm{N}->\mathrm{N}$ communication ; multicast will describe $1->\mathrm{N}$ communication and concentration the dual model $(\mathrm{N}->1)$. A set of peer entities using such means of communication will be called a group.

\subsection{Classic schemes of multipoint communication}

In a group of $\mathrm{N}$ processes, different schemes of communication may occur, according to the number of processes able to send messages. Figure 1 represents the basic schemes. 
In multicast communication a single process, called the sender, is likely to transmit messages to the other processes, called receivers.

When any members in a group of communicating processes can send messages to the others, we have a $\mathrm{N}->\mathrm{N}$ multipoint scheme : a message can be sent by any process, and will be delivered to the other active members of the group. There are examples of $\mathrm{N}->\mathrm{N}$ multipoint in [Navaratnam 88] and [Birman 91] .

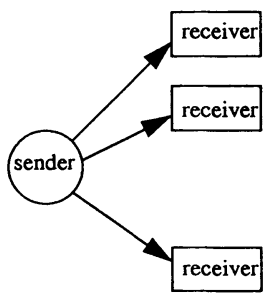

$1->\mathrm{N}$ multicast

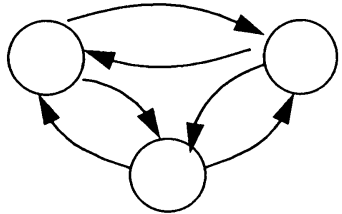

$\mathrm{N}->\mathrm{N}$ multipoint

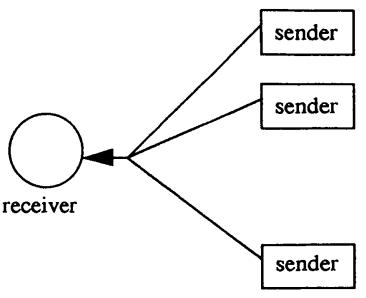

$\mathrm{N}->1$ concentration

Figure 1: schemes of multipoint communications

When each member of the group has to send the same kind of message to one receiver (acknowledgments, vote, ...), $\mathrm{N}$ messages will be delivered to the receiver. In order to limit the number of messages, it would be comfortable to collect them and to sum up information in a single message that will be delivered to the receiver's application. Figure 1 represents this concentration scheme.

In the following sections, we will focus on multicast communication. This choice can be justified for different reasons :

- In the same way it is possible to implement a 1 to $\mathrm{N}$ (multicast) conversation using $\mathrm{N}$ one to one (unicast) conversations, it is possible to decompose a " $\mathrm{N}$ to $\mathrm{N}$ " conversation into $\mathrm{N}$ multicast conversations ;

- the multipoint applications experimented today in the multimedia community use connectionless multicast communication support, and

- in the protocols studied below, there is none providing multipoint communication.

Finally, and because that is the way it is proposed in the protocols we have studied, we will regard the concentration ( $\mathrm{N}$ to 1 ) as a functionality of multicast where the concentration of information results from a multicast interrogation (see section 3.4.1).

\subsection{Some multicast protocols}

Multicast communication is supported by protocol functions. These protocols need to be implemented over a broadcast network, since no protocol provides a mechanism to broadcast 
packets on the physical network. Most of the LAN standards provide this facility (Ethernet, Token Ring, FDDI).

This section gives a global view of protocols designed for multicast communication. These protocols are mostly Transport level protocols. Thus, protocols of level three or lower, that mainly provide for multicast routing or for data broadcasting on a physical network are not studied in this paper. This explains, for example, why IP multicast [Deering 89] and ST II [Topol 90] are not discussed in this paper. Although not exhaustive, this list of protocols is representative of functionalities involved in multicast reliable transmission. For each protocol we briefly describe its origins, its functionalities and its design environment. References are given for more details. Some of the protocols we use to illustrate this analysis of reliability may seem old. In fact, recent protocols (designed in the past two years) do not introduce real new functionalities or mechanisms. We chose the following protocols because they introduced, at their time, novel ideas in multicast reliability. The most recent protocols will be analyzed in the conclusion of this paper.

- DANUBE is a Local Area Network developed at INRIA in 1981. A Transport protocol has been designed on this LAN to present an error detection mechanism in the transmission of messages from several sources to several destinations. The goal of this protocol was to match the performance of multipoint techniques with the reliability of end-to-end Transport mechanisms and to allow the definition of more efficient application protocols [Decitre 83].

- HLBC: This protocol [Leblanc 85] is implemented with the StarMod language [Cook 80]. StarMod executes on a network of eight Digital Equipment Corporation PDP 11/23 microprocessors connected by a carrier-sense (no collision detection) broadcast network. This protocol achieves a High-Level Broadcast Communication and offers improvements of up to 7.8 times over message transmission using peer-to-peer connections.

- Unix 4.2 MP [Ahamad 85] suggests a possible implementation of a Multicast Protocol (MP) on Unix 4.2 BSD [Joy 82], by generalizing the socket abstraction [Leffler 83] to include "multicast sockets". MP uses data structures available in the Unix kernel and takes advantage of both IP (Internet Protocol) and LNP (Local Network Protocol) protocols. This multicast experience under UNIX remains experimental, and can not be qualified as "multicast protocol" design. Moreover, Unix 4.2 MP is unreliable.

- VMTP: VMTP design (Versatile Message Transaction Protocol) is motivated to allow a strongly request/response oriented communication in a group of computers operating as a distributed system. VMTP is dedicated to Remote Procedure Calls (RPCs). It is connectionless and unreliable (even if it maintains enough information at the receiver's side to ensure ordered delivery of data received). Two implementations of VMTP exist : in the Unix kernel and in the $\mathrm{V}$ Distributed System, an experimental distributed operating system developed at Stanford [Cheriton 89].

- Multicast Protocol for Broadband Broadcast Networks (MPBBN) proposed by [Erramilli 87] attempts to be a reliable and efficient data transfer protocol for multicast applications. Its main objective is to achieve a throughput as independent of the size of the group as possible. This protocol operates in a broadband broadcast network, characterized by low error rate, low end- 
to-end delays, inexpensive transmission and storage.

- AMp and Turbo-AMp: Both multicast protocols have been designed for the Esprit Project DELTA4 (Definition and Design of an Open Dependable Distributed Architecture). They provide highly parallel reliable group communication primitives, which are useful in a number of applications such as client/server, distributed transactional and database systems, and fault tolerant techniques based on replicated computations. They are respectively working on token bus $(8802 / 4)$ and token ring (8802/5) networks [Verissimo 89] [Powell 91].

- XTP: XTP (Xpress Transfer Protocol) is a protocol designed by Protocol Engines Incorporated [PEI 92]. Its conception has been motivated by the needs of contemporary and future distributed, real-time, transactional and multimedia systems. Designed to be implemented in VLSI, XTP provides numerous services, including multicast communication, and is suited to Wide Area Networks characterized by significant transmission delays.

- GAM-T-103 is not a multicast protocol but a multicast Transfer Service. GAM-T-103 is a French military standard developed by Dassault Electronique for applications running in realtime static environments (system configured in its initialization phase). Multicast and concentration are provided by GAM-T-103 Transfer Service [ESD 89].

In the past five years new services and protocols that offer multicasting facilities have been designed. These are new generation protocols and services that use or adapt mechanisms described in this paper. These new developments will be described in the conclusion.

\section{MULTICAST RELIABILITY}

\subsection{Application requirements for multicast}

Applications that require multipoint communication service are numerous and diverse. To each of these applications corresponds a level of reliability that will be defined in the next sub-section. The most known multicast applications are:

- Resource location in distributed systems: During its execution, an application may need information unavailable on its site, or data whose particular location are unknown. This application distributes a request message to the other members of the group likely to keep the missing information in order to get it back ; at least one response is expected. Such a "request/ response" multicast is a good application for VMTP protocol. This type of multicast is used for transactions in a distributed database [Ahamad 88] or in distributed systems [Birman 87].

- Vote: When several processes have just finished the execution of the same application on different sites, a co-ordinating process collects their results to start a vote. Multicast is used to send that request (scheme $1->N$ ), while concentration is used to collect all the answers (see Delta4 [Powell 91]).

- Data replication: Replication is a simple way to achieve a crash-tolerant architecture. Active 
replication consists of executing the same application at different sites. The results are compared at the end of the execution with a vote. Obviously, applications have to work with the same environment on each site. Multicast is useful to update the same data on each site and to keep the sites in the same coherent state. That means updates have to be done on each site in the same order. Updating all copies of a passively replicated file or database also takes advantage of multicast.

- Teleconferencing : sending voice, video or data packets to all the members of a tele-conference [Aguilar 86]. These applications are today widely spread in the Internet world : IVS [Turletti 93] and NV [Frederick 92] are video-conferencing softwares ; WB [Jacobson 93] is a distributed white board that allows session participants to share (and modify) the same document at the same time. They are currently implemented over non reliable multicast support (UDP/IP), even if they require a different level of reliability.

- Video-On-Demand is merging most of the characteristiques of the previous systems. VOD systems provide the following functionalities : locate and access video information; manage distributed and replicated informations ; transmit pictures or video, and sound, using a clientserver type protocol. VOD will be in the close future, one of the most visible multicast application [Rowe 94][Deloddere 94].

These applications can justify the different level of multicast reliability described in the following section. The full range of multicast applications is much wider, for example multi-users games [Berglund 85] are another family of multicast applications.

\subsection{Reliability in multicast transmission}

Depending on the application, the required multicast communication support must be more or less sophisticated, and must provide different levels of reliability. For instance, multicast reliability may not be the same for resource location as for a database update. Moreover, the level of reliability depends on the control realized within the application (for example IVS, a videoconferencing application, provides its own flow control), and on the facilities provided by the underlying network. Different classes of multicast transmission have been defined to respond to the various levels of reliability.

\subsubsection{Connectionless multicast}

As in classic connectionless protocols, the sender only multicasts its packets, without worrying about their reception by the other group members. It is the easiest way to multicast information on a network. It requires that transmission control is realized directly by the application. The multicast protocol in Unix 4.2 is an example of connectionless multicast.

\subsubsection{K-reliable multicast}

Considering a group of $\mathrm{N}$ members, multicast will be called $\mathrm{k}$-reliable if at least $\mathrm{k}$ members of the group correctly receive the sender's message (with $0<=\mathrm{k}<=\mathrm{N}$ ). 1-reliable multicast is very often used when the sender does not need much control of data reception. For instance, 1reliable multicast is used for data location : it is not necessary to receive an answer from all 
group members, but at least one is needed. Multicast in VMTP [Cheriton 89] is 1-reliable. Danube and HLBC provide N-reliable multicast facilities. XTP provides both connectionless and reliable multicast. A typical application requirement for reliable multicast is storing copies of the same file on multiple file servers [Deswarte 90]. Note that connectionless multicast described in the previous section is equivalent to 0-reliable multicast.

\subsubsection{Atomic multicast}

Atomic multicast is considered to be $\mathrm{N}$-reliable multicast : in this kind of multicast, a message must be, either simultaneously delivered to all members of the group, or received by none of them. Atomic multicast is implemented in Delta4 in order to maintain the coherence of replicated data. HLBC is also an atomic protocol. A basic atomic multicast requires two phases :

- a data transfer phase (transmission / acknowledgment), as in point to point communication,

- a validation phase, to notify each receiver that the whole group received the data packets (Figure 2). Once a packet is validated, receivers are allowed to deliver the packets to the application. The validation phase does not exist in reliable non atomic protocols where data packets may be delivered as soon as received.

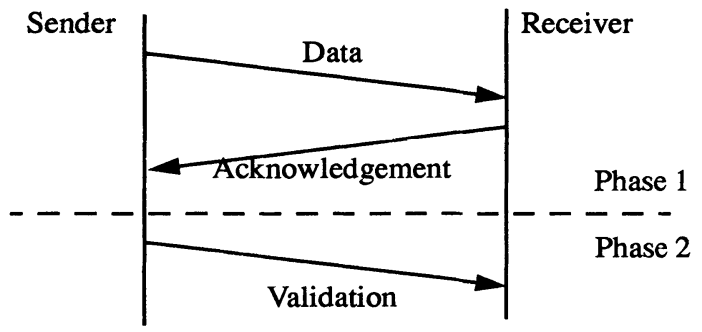

Figure 2: Two phases validation protocol

The two phases do not necessarily run in a stop-and-wait mode. The validation may be sent in an explicit packet, or implicitly carried by the next data transmission (see section 3.3).

\subsubsection{Ordering in reliable multicast}

In a group of $\mathrm{N}$ members, an ordered reliable multicast communication occurs when messages transmitted by a sender correctly reach all the receivers, and an ordering rule is verified between the receivers. Note that this order is not necessarily the transmission order, but the order in which data are delivered to the receiving protocol user (or application). In the literature, there are three different ordering notions to characterize a multipeer communication (names associated to these definitions may change following the author; the principle remain the same). 


\section{Partial ordering}

SDU received from each source are delivered in the same order at each receiver, but not necessarily in the order that they were transmitted by each source. There is no ordering rule specified between SDUs transmitted by different sources.

\section{Causal ordering}

SDU received from each source are delivered in the same order as the order of transmission by each source, with no ordering rule specified between Data Units (SDUs) transmitted by different sources.

\section{Total ordering}

All SDUs transmitted by any source are received and delivered in the same order at each receiver ; this order is the transmission order. Total order implies a global view of the connection, where data issued from different sources can be ordered.

Figure 3 summarizes these ordering notions. In the case of multicast, where there is only one sender and $\mathrm{N}$ receivers, causal and total ordering are equivalent. In the case of concentration, partial ordering corresponds to no ordering at all (that is why it is not represented).
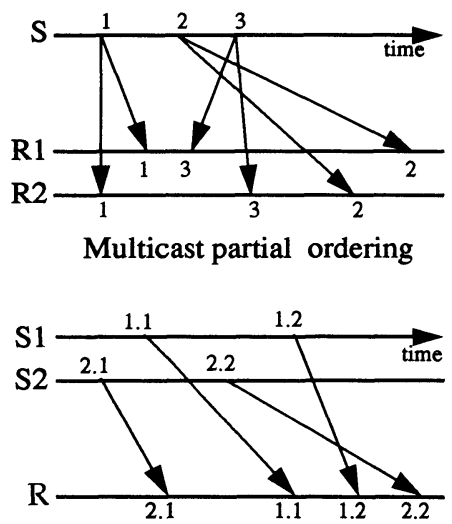

Concentration causal ordering
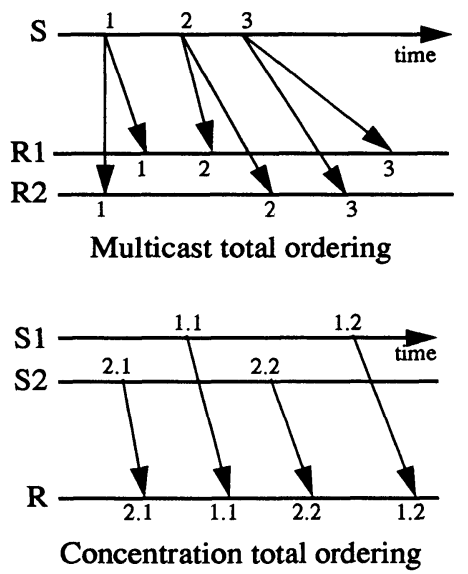

Figure 3 : Ordering in reliable multicast $(1->N)$ and concentration $(N->1)$

\section{CHARACTERISTIQUES OF RELIABLE MULTICAST}

In this section, we characterize reliable multicast protocols ( $\mathrm{N}$-reliable or atomic) according to their functionalities, and explain how the studied protocols provide reliability in multicast transmission. This analysis focuses on data transmission. Problems such as connection opening and closing are outside the scope of this paper. 


\subsection{Error control}

Messages may be lost or damaged during their transmission. Error control helps to detect, and sometimes, to correct errors. Two policies are available :

- positive acknowledgments : receivers inform the sender about received messages, or - negative acknowledgments : receivers inform the sender about corrupted or lost messages.

\section{Positive acknowledgment}

This kind of error control is classic in point-to-point Transport Layer protocols like TCP or OSI Transport class 4. In this scheme receivers have to send an explicit acknowledgment for each data packet or for a whole group of packets. If one acknowledgment is lost, the corresponding data packet(s) is(are) sent again. The main advantage of this solution, is that the sender regularly knows each receiver's state.

If the number of receivers is large, the network may be overloaded since each data message is acknowledged $\mathrm{N}$ times. Thus, one "useful" message induces up to $\mathrm{N}+1$ messages over the network $(\mathrm{N}+1$ when each data packet is acknowledged individually by each receiver).

Some experiments rely on positive acknowledgments. In Esprit project Delta4, this kind of control has been implemented on lower OSI layers (MAC layer for Turbo-AMp, LLC layer for $\mathrm{AMp}$ ). [LeBlanc 85] relates interesting results over $\mathrm{N}$ point-to-point communications.

\section{Negative acknowledgment}

On the sender side, negative acknowledgment error control consists of numbering outgoing data packets. On the receiver side, it consists of detecting sequence gaps.

For instance, if a receiver gets the data packet sequence \#1, \#2, \#3 and \#5, it will notify the sender of the missing packet \#4. If this acknowledgment is lost, it will be sent again when the receiver sees \#6, \#7... Receiving the request for message \#4, the sender will retransmit the specified packet using either a selective retransmission strategy or a Go-back- $n$ retransmission (the retransmission starts again from the first missing packet detected).

This idea was first applied to multicast by DANUBE and MPBBN. It was then used by many protocols such as XTP. Its main advantage is limiting traffic over a reliable network. But negative acknowledgment works only in a continuous data stream context. Moreover, receivers do not notify the sender of their state until they have received a damaged packet, or an out-oforder packet. A receiver may have crashed or lost all the last data packets without the sender getting any knowledge of this. This is critical in atomic multicast protocols where only messages received by everyone are validated and delivered.

\section{Mixed solution}

A reliable solution comes from mixing negative and positive acknowledgments (XTP and MPBBN) : an acknowledgment is sent back on sender request, or spontaneously by the receiver when a packet loss is detected. XTP introduces an optimization over simple negative acknowledgments : the slotting / damping. With slotting, acknowledgment transmission is delayed by a random delay. Its first effect is to avoid an acknowledgment burst to the sender on 
acknowledgment request. With damping, acknowledgments are multicasted to the whole group (including the sender), allowing receivers to identify and dequeue redundant error control messages. For example, suppose that $\mathrm{R} 1$ receives a request for packet \#4 retransmission from receiver $R 2$. It will not send its request for packet \#6 because the negative acknowledgment from $R 2$ means that packets \#4, \#5, \#6 ... will be sent again on $R 2$ request. The main drawback of damping only is that it induces significant overhead for receivers as they have to process acknowledgments too.

\subsection{Flow control}

When a sender transmits faster (or is less loaded) than the receivers, it will systematically transmit more packets than receivers can accept. Even if no error occurs during the transmission, the receivers can not process all data packets and will lose some of them. A flow control mechanism matches sender throughput with the receiver's throughput. We have observed two cases:

- Stop and Wait based protocols : the sender stops and waits for acknowledgments after sending each data packet or a group of data packet. This scheme is used in Delta4 and HLBC;

- Window based protocols : (some data packets may be sent before any acknowledgment comes back : XTP, MPBBN, DANUBE ...). This second case may be managed in two ways :

- receiver spontaneous acknowledgments ; or

- acknowledgments on sender request.

\section{Stop and Wait protocols}

In this context, flow control is quite simple :

- In HLBC, if the sender does not receive all acknowledgments, it can send its data again up to $\mathrm{K}$ times. Over this limit, the sender will consider the transmission has failed.

- In Delta 4, receivers may notify the sender of buffer overflow with a "bad_reception" message. Then, the validation phase will throw data packets away.

\section{Window based protocols : receiver's spontaneous acknowledgments.}

If error rates are low and round trip delays are small, protocols may use negative acknowledgment error control. Most of the time, negative acknowledgments are mixed with positive acknowledgments or timers. The sender manages a $\mathrm{K}$ data packet wide window (data packets numbered from $\mathrm{k}$ to $\mathrm{k}+\mathrm{K}-1$ ) and a moving cursor pointing to the next data packet to be sent.

This kind of flow control is used in MPBBN and DANUBE. Three cases may occur :

1. Reception of a negative acknowledgment about data of rank $n$ ( $n$ is inside the window): the cursor moves back to data of rank $n$.

2. Reception of all positive acknowledgments about data of rank $n$ : the window slides to start from rank $n+1$ and the cursor points to rank $n+1$ too. 
3. The last data in the window has been transmitted. The cursor now points to data of rank $\mathrm{k}+\mathrm{K}$. At least $\mathrm{K}$ chances have been offered to the receivers to recover from the loss of the data packet of rank $\mathrm{k}$ and the window shifts one data packet forward ( $\mathrm{K}$ is a parameter which is related to the probability of packet loss, the expected burstiness of the data traffic and the network delay: it is supposed to give a receiver enough opportunities to send a negative acknowledgment and the sender to receive it). If they have not, they will be considered as failed.

\section{Window based protocols : acknowledgments on the sender's request.}

The mechanism described corresponds to XTP flow control mechanism, which is referred to as the "bucket algorithm". An XTP sender requests acknowledgment packets each interval ST. ST depends on the measured round trip time on the connection; its duration is based on the amount of time an XTP sender waits for a response to an acknowledgment request. For each acknowledgment request transmitted, the sender creates a bucket that collects corresponding acknowledgments. Buckets are numbered; each acknowledgment refers to a bucket number.

Acknowledgments carry flow control information (that allow, for instance, sliding the sending window) and error control information (retransmission request). The sender needs at least one flow control message from each receiver because it cannot slide its window without the agreement of all receivers: That is why the algorithm keeps in mind the B youngest buckets. $B$ is sized to give enough time and opportunities for a receiver to have at least one of its acknowledgments in one of the B buckets despite request / acknowledgment round trip time and network failures. If it has not, the receiver will be considered as crashed.

Each ST, the sender scans the B buckets and starts packet retransmission to the lowest rank over all buckets. That means acknowledgments are processed only each ST, which delays data retransmission. This allows the sender to receive the slowest receiver's acknowledgments in order to process only one retransmission.

ST must be large enough to not overload the network with control packets, but it must be small enough to make the interval B*ST not too large : assuming the network is reliable, the bucket algorithm will always start retransmission with the information contained in the oldest bucket. Younger packets will be re-sent although they have already been received by the receivers, creating non required retransmission.

The specifications of XTP do not exclude rate control in multicast, but it is not yet implemented in the bucket algorithm.

\subsection{Validation}

The validation phase is required for atomic multicast only. It may be achieved with an explicit validation packet (Delta4) or in the next data packet (HLBC).

\section{Explicit validation (Delta4)}

The Delta4 multicast protocol, AMp is implemented over layer two (MAC level) of the OSI stack. It allows the multicast of one message (in contrast to a group of messages) in an atomic way. To ensure atomicity, the sender may send one of the following : 
- a validation packet if all members have correctly acknowledged the corresponding data packet;

- a reject packet if one member has not, or if delivery conditions are not verified.

If lost, the validation packet is requested by the appropriate receivers.

\section{Validation carried by data packets}

HLBC uses an alternated bit scheme error control. It waits for all acknowledgments about the current data before sending new data. New data reception means that previous data can be delivered to the application. Validation carried out by data packets may be applied to data stream oriented protocols like MPBBN, DANUBE and XTP with minimal change. Two solutions are possible:

- If the receiver knows the size $B$ of the sender window, the reception of a data packet with rank $n$ validates any data packet with rank less than $n-B$. This solution involves a significant delay between the reception and the delivery of a packet. Moreover, some events, like the sliding of the sender window on reception of positive requested acknowledgments, are not taken into account.

- A field including validated packet rank may be added to the control part of data packets. This solution does not charge the network with extra packets.

\subsection{Other characteristics}

This section introduces characteristics we did not use to classify multicast reliability : concentration, group management policy and routing problems (multicast in WANs). These functionalities, that are typical from multicast protocols, are not directly linked to reliability, but can have important side effects the way reliability can be guaranteed. They are only briefly reviewed in this paper.

\subsubsection{Concentration}

Concentration ( $\mathrm{N}->1$ communication) is the dual scheme of multicast. It consists of summarizing $\mathrm{N}$ messages to the destination application. In order to reduce the traffic on the network, one may want to concentrate messages as soon as possible. An immediate application of concentration for multicast protocols is positive acknowledgment collection.

Turbo-AMp, which is a more sophisticated implementation of AMp (Delta4) provides facilities for concentration through acknowledgment collection. It takes advantage of the network topology and aims at exploiting some specific details of the token-ring MAC protocol ${ }^{1}$. In this protocol, sites are distributed on a ring and, before being delivered to their destination, messages have to cross encountered sites on the ring. When transmitted, data have to go around the ring, in order to reach any potential receiver, before coming back to the sender. Turbo-AMp uses a technique called "acknowledgment in flight" : a space is reserved in data packets to collect acknowledgments. When a site receives data destined to one of its users, it mixes its posi-

1.Concentration in Wide Area Networks is theoritically possible at the interconnection level ; this implies the existence of multicast protocols at the Network Layer working in connection oriented mode. 
tive acknowledgment (or its negative acknowledgment if, for instance, the reception buffer is full) with the acknowledgment on the data packet, using a simple boolean function. In a favorable case, only one single message is needed for the whole sending/reception phase, independent of the number $\mathrm{N}$ of receivers. This protocol implies a hardware modification on the tokenring, which cannot be realized for commercial implementations.

GAM_T 103 provides for concentration in a different manner. Concentration is associated with a connection model called "centralized connection with receiver as master", where the concentration step only occurs after a "request" message has been multicast by the master to the other participants of the connection. This functioning is mostly justified by application requirements. In other words, GAM_T 103 only addresses applications where concentration results from an information request that has been previously multicasted.

\subsubsection{Group management}

\section{Group structure}

A group is defined as the set of entities involved in a multipoint communication. Entities involved in a group are called members. Different levels of definition exist for a group :

- A group can define all the entities that can participate in a multipeer conversation. For example, all the people that have an Internet address can participate in a video-conferencing session. These people form a group.

- Within this group, a sub-group can be defined as those that want to participate in a particular multipoint conversation. To belong to this sub-group, an entity has first to be in the higher level group, and then to explicitly apply for participation to the conversation (this action is generally called a group registration).

- While registered in the group of entities that participate in a multipeer conversation, an entity can either be active or inactive. This defines an other sub-level of group. An active member can contribute to the conversation ; a passive member cannot. But during a multipoint connection, a member has to be able to switch easily from active to passive, and vice-versa. A member that becomes active (resp. passive) joins (resp. leaves) the active group.

\section{Group integrity}

Behind the notion of active group, the notion of group integrity can be defined. The integrity of a group is defined by a condition which can specify a minimum number of participants, or explicitly give the address of entities that must participate in the conversation. The Active Group Integrity (or AGI) Condition can also specify which of the members can transmit, and which of them can only receive.

XTP and Delta4 have two opposite policies in the way they manage groups. The XTP sender need not know the identity nor the number of the receivers. XTP considers that $B$ buckets are sufficient to collect the acknowledgments from the whole group. If a receiver is not identified in the B buckets, it is considered failed or not belonging to the group. Because of its validation strategy, a Delta4 sender needs to know at least the number of its receivers to be sure to collect all the acknowledgments. This difference allows a simpler connection management for XTP. 


\subsubsection{Multicast in WANs}

Multicast is well supported by Local Area Networks conforming to the IEEE 802 standards which provides broadcast delivery and multicast addresses [IEEE 85]. However, implementing multicast on a Wide Area Network is further complicated because of routing problems. For example, consider the situation of figure 4 in which the members of a multicast group are located on two different networks A and B interconnected by a gateway. Sending a unicast message is quite easy since the gateway forwards it to the remote destination, while multicasting a message raises problems since the message has to be transmitted on both the local and remote network :

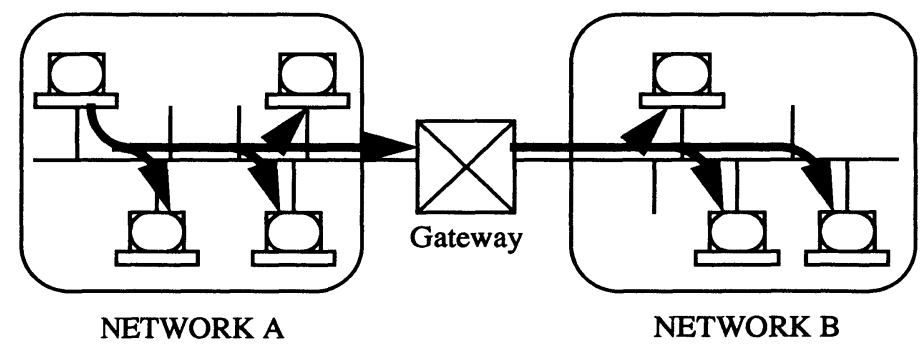

Figure 9: Multicast group located on two LANs

- Destination identification may differ between both networks so that it is necessary to maintain a list of the different remote destination identifiers.

- If the sender manages this list, it may send to the gateway:

- as many messages as individual remote receiver, or

- a single message and the list of remote receivers (the gateway will then be charged to multicast the messages to the remote receivers).

- If the gateway manages the list, the problem is the way the sender indicates that it wants to multicast a message to remote receivers of the group.

[Hughes 87] examines different methods of inter-network multicast communications well suited to different designs of gateways. [Deering 90] describes different routing algorithms commonly used by link-layer bridges to reduce the costs of multicast in large extended LANs. [Tode 92] proposes two multicast routing algorithms which aims at approximating solutions for a minimum cost path when multicasting packets through nodes of the networks.

A lot of algorithms are proposed in the literature, but few of them have been successfully implemented. Consequently, few multicast protocols are working on WANs: the Unix 4.2 multicast protocol allows to send messages to remote networks using the Internet Protocol (IP) and existing data structures of Unix (generalization of the concept of sockets). XTP seems to be well suited to use multicast on WANs, but the routing part is not entirely defined yet. 


\section{CONCLUSION}

This paper has presented a survey of reliability in multicast protocols and of associated mechanisms. In table 1, these protocols are summarized with respect to the mechanisms analyzed in the paper. The first part of this table presents the reliable multicast protocols we have studied. In the second part, there are two unreliable multicast protocols given for comparison purposes. GAM-T-103 is isolated in the last part of the table because it is not a protocol, but a service.

Table1: Synthesis of the characteristics of the studied multicast protocols.

\begin{tabular}{|c|c|c|c|c|c|c|}
\hline Protocol & \begin{tabular}{llll}
\multicolumn{4}{c}{ Layer } \\
2 & 3 & 4 & 5
\end{tabular} & Reliability & $\begin{array}{c}\text { Error } \\
\text { control }\end{array}$ & $\begin{array}{l}\text { Flow } \\
\text { control }\end{array}$ & Validation & $\begin{array}{l}\text { Concen } \\
\text { tration }\end{array}$ \\
\hline XTP & $\mathbf{X}_{\mathbf{1}}^{\prime} \mathbf{X}_{\mathbf{1}}^{\prime}$ & cls/con & Damping & Wind./Rate & No & \\
\hline DANUBE & $: X_{1}^{\prime}$ & con & Nack & Window & No & \\
\hline MPBBN & $\mathbf{x}$ & con & Nack & Window & No & \\
\hline HLBC & ' $x$ & Atomic & Ack & Stop\&Wait & with data & \\
\hline AMp & $\begin{array}{l:l} \\
\mathbf{x}\end{array}$ & Atomic & Ack & Stop\&Wait & Explicit & \\
\hline Turbo AMp & \begin{tabular}{|l:l}
$X_{1}^{\prime}$ & $:$
\end{tabular} & Atomic & Ack & Stop\&Wait & Explicit & $\mathbf{X}$ \\
\hline
\end{tabular}

\begin{tabular}{|c|c|c|c|c|c|c|c|}
\hline VMTP & & $\mathbf{X}$ & 1-reliable & Piggyback & No & No & \\
\hline UNIX 4.2 & & $\vdots X$ & cls & No & No & No & \\
\hline
\end{tabular}

\begin{tabular}{|l|l|l|l|l|l|l|}
\hline GAM-T 103 & $\mathbf{X}: \mathbf{X}_{\mathbf{1}}$ & con & Ack & Window & No & $\mathbf{X}$ \\
\hline
\end{tabular}

In the past two years, new results on multicasting have appeared, contributing new Transport protocols and service definitions. The purpose of these new protocols is not to address reliability in multicast transmission. The last major contribution on this subject comes from XTP, which was the first general purpose protocol to provide for multicasting. The new protocols address other problems of group communication which are directly linked to multimedia application requirements. They mostly focus on (but are not limited to) group addressing, Quality of Service policy, and synchronisation (respect of the time constraints). This explains why these protocols are not analyzed in the previous survey. Examples of these new development are :

- The TENET group, in ICSI Berkeley, designs and experiments with new communication systems [Ferrari 92a] [Ferrari 92b] for multimedia environments and high performance network.

- ST II [Topol 90] is an IP-layer protocol that provides end-to-end guaranteed service across an internet (but is not originally an Internet work). ST II is intended to support and facilitate the implementation of a wider range of applications than IP. 
- The ISO has started the project "Enhanced Communication Functions and Facilities (ECFF)" [ISO-SC6 93a] to add multipeer services for real time, voice and video transmission to the OSI stack, and to improve transport layer efficiency and flexibility. The notion of efficiency considers both implementation and new services. New protocols and services have been proposed within the ECFF project :

- The High Speed Transport Protocol and Service (HSTP and HSTS) based on XTP.

- The TPX (Transport Protocol class X) [Danthine 92] and its associated transport service, a new high performance transport layer designed in the ESPRIT European Project OSI 95.

- ETS, the Enhanced Transport Service [Diot 92] based on GAM-T 103 transfer service. It is a service designed specifically for multipeer real time applications.

- European project CIO contributed with the proposal of enhanced protocol facilities for multicast [Milou 93].

The ISO, in its last plenary session, admitted that an enhanced communication stack has to be designed considering application requirements. A reflection on services to be introduced at the transport level started under the name ECTS (for Enhanced Communication Transport Services). A multicast taxonomy document is actually under definition [ISO-SC6 93b].

- The Internet is more active on multicasting protocol mechanisms at the Network level. The IDMR project copes with Inter Domain Multicast Routing [Moy 91][Ballardie 93] . But today, the most visible activity is the modification of IP to extend its addressing field and to offer enhanced mechanisms (including multicast capabilities) [Katz 93] [Deering 93]. At the Transport level, some work intends to extend the TCP protocol with enhanced capabilities [Braden 92][Jacobson 92] ; RTP is a multicast protocol that defines a header format [Schulz 93] (not a full protocol specification). RTP has the status of Internet draft.

These new developments follow a different approach from the one used in the past : new protocols and services are designed considering the application requirements, and no longer considering only the facilities provided by the underlying network. This "Top-Down" approach is now generalized and we can expect, in coming years, major improvements in the ways communication systems provide guaranteed services to applications.

\section{ACKNOWLEDGMENTS}

The author wants to thank those who contributed to this paper, by pro-iding ideas, comments, and editing corrections. With particular thanks to:

- Sébastien BOUAT and Patrick SCABELLO, students at the ENSIMAG. This paper relates their work on Multicast.

- Larry GREEN (XTP Forum) and Christian HUITEMA (INRIA) who introduced valuable ideas in the discussion, and in the elaboration, of this paper. 


\section{REFERENCES}

[Aguilar 86] L. Aguilar, J. Garcia-Luna-Aceves, D. Moran, E. Craighill, R. Brungardt, Architecture for a Multimedia Teleconferencing System, ACM 1986 Symposium on Communications Architectures and Protocols, Stowe,Vermont,pp 126-135, Aug. 1986.

[Ahamad 85] M. Ahamad, A. Bernstein, Multicast Communication in Unix 4.2bsd, IEEE Computer Society, May 1985,pp 80-87.

[Ahamad 88] M. Ahamad, M. H. Ammar, J. M. Bernabeau-Arban, M. Khalidi, Using Multicast Communication to Locate Ressources in LAN-Based Distributed System, Proceedings of the 13th Conference on Local Computer Networks, IEEE, Mineapolis, Minnesota, 1988.

[Ballardie 93] T. Ballardie, Core based Trees (CBT). An Architecture for Scalable InterDomain Multicast Routing, SIGCOM '93. September 13-17, 1993, San Francisco (USA).

[Berglund 85] J.Berglund, D. Cheriton, Amaze:A Multiplayer Computer Game, IEEE Software, Volume 2, No 3, May 1985.

[Birman 87] K. Birman, T. Joseph, Reliable Communication in the Presence of Failures, ACM Transactions on Computer Systems, Vol.5, No1, February 1987, pp 47-76.

[Birman 91] K. Birman, A. Schiper, P. Stephenson, Lightweight Causal and Atomic Group Multicast, ACM Transactions on Computer Systems, Vol.9, No 3, Aug. 1991, pp 272-314.

[Braden 92] R. Braden, Extending TCP for Transactions -- Concepts, RFC 1379. 38 p. November 1992.

[Cheriton 89] D. R. Cheriton, C. L. Williamson, VMTP as the Transport Layer for High-Performance Distributed Systems, IEEE Communications Magazine, June 1989.

[Cook 80] R. P. Cook, MOD - A Language for Distributed Computing, IEEE Transactions on Software Engineering, vol. SE-6, No 6, Nov 1980, pp248-253.

[Danthine 92] A. Danthine, A New Transport Protocol for The Broadband Environment. IFIP Workshop on Broadband Communications, Estoril, 20-22 January 1992.

[Decitre 83] P. Decitre, A. Khider, G. Vandome, Protocole de diffusion fiable pour réseaux locaux, IMAG report RR N 347, Jan.1983.

[Deering 89] S. Deering, Host Extensions for IP Multicasting. RFC 1112. 17. August 1989.

[Deering 90] S. Deering, D. Cheriton, Multicast Routing in Datagram Internetworks and Extended LANs, ACM Transactions on Computer Systems, Vol.8, No 2, May 1990, pp 85-110.

[Deering 93] S. Deering, SIP : Simple Internet Protocol, IEEE Networks. May 1993. 
[Deloddere 94] D. Deloddere, W. Verbiest, H. Verhille, Interactive Video on Demand, IEEE Communication Magazine, May 1994.

[Deswarte 90] Y. Deswarte, L. Blain, J. M. Pons, Delta4 Architecture Guide: Security, LAAS Reference: 90321, Delta4 Reference G90.066/D3/C

[Diot 92] C. Diot, P. Cocquet, D. Stunault. ETS, the Enhanced Transport Service. Research Report RR 907 I. IMAG Institute. Grenoble. May 1992.

[Erramilli 87] A. Erramilli, R. P. Singh, A reliable and efficient multicast protocol for broadband broadcast networks, SIGCOMM'87 Workshop, ACM, August 1987.

[ESD 89] Electronique Serge Dassault, Réseaux Locaux Temps Réel Militaires. Protocoles Couche Transfert Recital. GAM-T-103-C, March 1989.

[Ferrari 92a] D. Ferrari, A. Banerjea, H. Zhang, Network Support for Multimedia, a Discussion of the TENET Approach. Technical Report TR-92-001. ICSI, UC Berkeley. November 1992.

[Ferrari 92b] D. Ferrari, J. Ramaekers, G. Ventre. Client-Network Interactions in Quality of Service Communication Environments. Proceedings of the fourth IFIP International Conference on High Performance Networking (HPN '92). Liège. December 1992.

[Frederick 92] R.Frederick, $N V$ - XI1 video-conferencing tool, Unix Manual Page, XEROX PARC, 1992.

[Hughes 87] L. Hughes, Multicast Gateway Design Considerations, Computing Laboratory, University of Newcastle upon Tyne,1987.

[IEEE 85] IEEE Computer Society. Standards for local area networks: Logical link control. ANSI/IEEE Standard 802.2- 1985 (ISO/DIS 8802/2), 1985.

[ISO-SC6 92] USA - ANSI contribution on Multipeer Data Transmission Transport Service. ISO/IEC JTC1/SC6 N 7445. August 28, 1992.

[ISO-SC6 93a] Revised Scope and Purpose and Justification for ECTS Project. ISO/IEC JTC1/SC6/WG4 4K74. Seoul. October 15, 1993.

[ISO-SC6 93b] Draft Multicast Taxonomy of Multicast Operation. ISO/IEC JTC1/SC6/WG4 N 833. October 31, 1993.

[Jacobson 92] V. Jacobson, R. Braden, D. Borman. TCP extensions for High Performance. RFC 1323. 37 p. May 1992.

[Jacobson 93] V. Jacobson. WB README file. Lawrence Berkeley Laboratory (LBL). August 1993.

[Joy 82] W. Joy, E. Cooper, R. Fabry, S. Leffler, M. McKudick, 4.2bsd Systems Manual, Com- 
puter Systems Research Group, Technical Report 5, University of California, Berkeley.

[Katz 93] D. Katz, P. Ford. TUBA : replacing IP with CLNP. IEEE Networks. May 1993.

[LeBlanc 85] T. J. LeBlanc, R. P. Cook, High-Level Broadcast Communication for Local Area Networks, IEEE Software, May 1985, pp 40 - 48.

[Leffler 83] S. Leffler, W. Joy, R. Fabry, 4.2 bsd Interprocess Communication Primer, Computer Systems Research Group, University of California, Berkeley, July 1983.

[Milou 93] I. Miloucheva. Specification of Enhanced Protocol Facilities for Multicast and Broadcast (M26b). Technical report from RACE project 2060 (CIO). October 16, 1993.

[Moy 91] J. Moy, OSFP version 2, Internet RFC 1247, 189 p., July 1991.

[Navaratnam 88] S. Navaratnam, S. Chanson, G. Neufeld, Reliable Group Communication in Distributed Systems, IEEE Computer Society, June 1988, 439-446.

[PEI 92] Protocol Engines Incorporated, XTP Protocol Definition, revision 3.6, jan. 1992.

[Powell 91] David Powell, Delta4: A generic Architecture for Dependable Distributed Computing, Research Reports ESPRIT, 484 p., ISBN N3-540-54985-4, Springer-Verlag, Berlin,Germany, 1991.

[Rowe 94] L. A. Rowe, Berkeley Video-On-Demand Server, presentation transparencies, May 1994 (papers/BerkeleyVOD-Talk.ps.Z).

[Schulz 93] H. Schulzrinne, S. Casner, RTP : A Transport Protocol for Real-Time Applications, Internet Draft of the Audio-Video Working Group (draft-ietf-avt-rtp-04.ps), IETF, October 1993.

[Tode 92] H. Tode, Y.Sakai, M.Yamamoto, H.Okada, Y.Tezuka, Multicast Routing Algorithm for Nodal Load Balancing, IEEE Infocom92, Florence,Italy, May 1992.

[Topol 90] C. Topolcic, Experimental Internet Stream Protocol, Version 2 (ST II), Internet RFC 1190. October 1990.

[Turletti 93] T. Turletti. H 261 Software Codec for Videoconferencing over the internet. Research Report N. 1834. INRIA Sophia Antipolis. January 1993.

[Verissimo 89] P. Verissimo, L. Rodrigues, M. Baptista, AMp : A highly parallel atomic multicast protocol, Computer Communication Review, Vol.19, No 4, Sept. 89, pp 83-93. 